\title{
Journal of Applied Glycoscience Vol. 62 No. 4 (November 2015)
}

\section{- Regular Papers}

Satoshi Maeda, Yuta Kazama, Atsushi Kobayashi, Akira Yamazaki, Sumiko Nakamura,

Masayuki Yamaguchi, Hideo Maeda, and Ken'ichi Ohtsubo

Improvement of Palatability and Prevention of Abrupt Increases in Postprandial Blood Glucose Levels by Hokurikukona243 after High Pressure Treatment 127

Tomonari Tanaka, Atsushi Tsutsui, Ryuya Gotanda, Shota Sasayama,

Kazuya Yamamoto, and Jun-ichi Kadokawa

Synthesis of Amylose-polyether Inclusion Supramolecular Polymers by Vine-twining Polymerization Using Maltoheptaose-functionalized Poly(tetrahydrofuran) as a Primer-guest Conjugate 135

Yayoi Miyagawa, Masashi Yoshino, Takashi Kobayashi, and Shuji Adachi

Kinetic Analysis for the Conversion of Fructose to 5-Hydroxymethylfurfural in 1-Butyl-3-methylimidazolium Chloride with Lower Water Contents 143

\section{-Notes}

Kazuhiro Nara and Yoji Kato

Structural Characterization of a Heterogalactan from Antler-shaped Ganoderma lucidum

149

Takako Hirano, Rie Uehara, Haruka Shiraishi, Wataru Hakamata, and Toshiyuki Nishio

Chitin Oligosaccharide Deacetylase from Shewanella woodyi ATCC51908 153

Takahiro Noda, Shigenobu Takigawa, Chie Matsuura-Endo, Koji Ishiguro,

Koichi Nagasawa, and Masahiro Jinno

Properties of Calcium-fortified Potato Starch Prepared by Immersion in Natural Mineral Water and Its Food Application

159

\section{-Appendix}

Table of Contents Vol. 62 (2015) 165

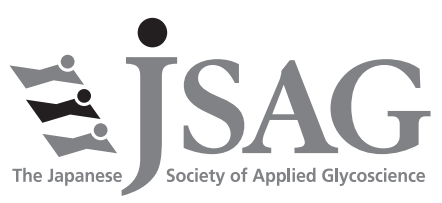

WEB $\gg \gg \gg \gg \gg \gg$

J-STAGE

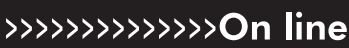

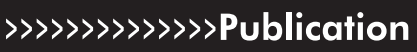

http://www.jstage.jst.go.jp/browse/jag 